\title{
Functional interaction of BRCA1/ATM-associated BAAT1 with the DNA-PK catalytic subunit
}

\author{
EUI YOUNG SO and TORU OUCHI \\ Department of Medicine, NUHS, Systems Biology Program, Pritzker School of Medicine, \\ The University of Chicago, Evanston, IL 60201, USA \\ Received December 1, 2010; Accepted March 2, 2011
}

DOI: $10.3892 /$ etm.2011.232

\begin{abstract}
Ataxia telangiectasia mutated (ATM) and DNA-dependent protein kinase (DNA-PK) play a crucial role in the initial stages of cell response, when cells are exposed to DNA insult such as ionizing radiation (IR) and chemical agents. We previously demonstrated that ATM requires BAAT1 for its activation in response to IR. In the present study, BAAT1 was found to bind to the DNA-PK catalytic subunit (DNA-PKcs) and SMC1. Biochemical analysis indicated that several regions of BAAT1 were responsible for the interaction with these proteins, and their binding affinity was altered after treatment with the IR mimetic, neocarzinostatin (NCS). Phosphorylation of the DNA-PKcs at Ser2056 and SMC1 at Ser966 was induced by NCS, while phosphorylation was reduced when BAAT1 was depleted by siRNA. These results indicate that BAAT1 globally regulates DNA damage signaling during the early stages of apoptosis.
\end{abstract}

\section{Introduction}

Cells must ensure that their genetic information is accurately transmitted to each daughter cell during the cell division process. To ensure that the DNA has been fully replicated in an undamaged state, cells progress through a series of cell cycle checkpoints prior to mitosis. Incomplete DNA replication due to stalled replication forks, DNA damage caused by ultraviolet light, chemicals in the environment, gamma radiation or reactive oxygen species, or treatment with chemotherapeutic agents used in cancer therapy activate checkpoint signaling pathways that arrest the cell cycle, providing damaged cells time to complete DNA synthesis or to repair DNA damage. If the DNA is too severely damaged, alternate signaling pathways are activated, leading to permanent growth arrest or programmed cell death (1).

Correspondence to: Dr Toru Ouchi, NUHS, Systems Biology Program, Pritzker School of Medicine, The University of Chicago, 1001 University Place, Evanston, IL 60201, USA

E-mail: touchi@bsd.uchicago.edu

Key words: BAAT1, DNA-dependent protein kinase catalytic subunit, DNA damage
Carcinogenesis is often caused by a functional mutation or the loss of proteins involved in cell cycle checkpoints, which provides tumor cells with the selective advantage of proliferation. Defects in cell cycle checkpoints are the hallmarks of tumor cells, and also determine the efficacy of cancer treatment with anti-neoplastic agents. Chemotherapeutic agents and IR usually damage the DNA of both normal and tumor cells. Normal cells activate cell cycle checkpoints, leading to growth arrest and subsequently the DNA repair process. In tumor cells, however, the loss of appropriate checkpoints due to mutations in proteins such as p53, Chk1 or Chk2 allows malignant cells to progress to mitosis with numerous mutations of single- and double-strand breaks (DSBs) still present in their genomic DNA. As a result, mutations in the genome accumulate progressively, leading to apoptosis (2).

Mechanisms of sensing and repairing damaged DNA are conserved among the species. Ataxia telangiectasia mutated (ATM) and the catalytic subunit of DNA-dependent protein kinase (DNA-PKcs) are essential to the DNA damage response when cells are exposed to DNA insult as described above. Activation of these kinases has been implicated in the initiation of the DNA repair process and cell cycle checkpoints. By phosphorylation of their substrates, these kinases transmit signals to downstream proteins, including the Mre11/ Rad50/Nbs1 (MRN) complex, Fanconi anemia proteins and the BRCA1 breast cancer tumor-susceptibility protein (3-6).

We recently identified a novel protein, named BAAT1 (BRCA1-associated protein required for ATM activator-1) (7), which was isolated by yeast two-hybrid screening using aa1314-1863 of BRCA1 as bait. We found that BAAT1 also binds to ATM, localizes to DSBs and is required for Ser1981 phosphorylation of ATM under conditions of IR treatment. siRNA-mediated stable or transient depletion of BAAT1 was found to result in decreased phosphorylation of ATM at Ser1981, resulting in impaired phosphorylation of NBS1, Chk2 and $\gamma \mathrm{H} 2 \mathrm{AX}$ after IR. Treatment of BAAT1-depleted cells with okadaic acid greatly restored the phosphorylation of ATM Ser1981, suggesting that BAAT1 is involved in the regulation of ATM phosphatase. PP2A-mediated dephosphorylation of ATM was partially blocked by purified BAAT1 in vitro. These results suggest that DNA damage-induced ATM activation requires a coordinated assembly of BRCA1, BAAT1 and ATM.

In the present study, we detail the functional interaction of BAAT1 with the proteins involved in the DNA damage 
response. The results demonstrate that the DNA-PKcs also requires BAAT1 for its activation after DNA stress. These results suggest that BAAT1 is commonly required for the initial stage of the DNA damage pathway.

\section{Materials and methods}

Cell culture. The human embryonic kidney 293T cell line was grown in Dulbecco's modified Eagle's medium (DMEM) (Invitrogen, Carlsbad, CA) containing 10\% fetal bovine serum (FBS; Invitrogen), penicillin/streptomycin and $10 \mathrm{mM}$ sodium pyruvate (Sigma, St. Louis, MO). MCF10A cells were cultured on tissue culture flask (Corning) in a 1:1 mixture of DMEM and F12 medium (DMEM-F12) supplemented with $5 \%$ horse serum, hydrocortisone $(0.5 \mu \mathrm{g} / \mathrm{ml})$, insulin $(10 \mu \mathrm{g} /$ $\mathrm{ml})$, epidermal growth factor $(20 \mathrm{ng} / \mathrm{ml})$ and penicillinstreptomycin $(100 \mu \mathrm{g} / \mathrm{ml}$ each).

Immunoblot analysis and immunoprecipitation. Cells were treated with the IR mimetic neocarzinostatin (NCS) $(0.5 \mathrm{mg}$ / $\mathrm{ml}$ ) for the indicated times and then lysed in ice-cold lysis buffer [(50 mM Tris- $\mathrm{HCl}(\mathrm{pH} 7.6), 150 \mathrm{mM} \mathrm{NaCl}, 1 \mathrm{mM}$ EDTA (pH 8.0), $20 \mathrm{mM} \mathrm{NaF}, 1 \mathrm{mM} \mathrm{Na} \mathrm{VO}_{4}, 1 \% \mathrm{NP}-40$, $0.5 \mathrm{mM}$ dithiothreitol] in the presence of protease-inhibitor mix [leupeptin, aprotinin and phenylmethylsulfonyl fluoride (PMSF) $(10 \mu \mathrm{g} / \mathrm{ml})$, respectively]. After centrifugation $(12,000 \mathrm{x} \mathrm{g}, 10 \mathrm{~min})$, soluble supernatants were prepared, and the protein concentrations were calculated using the Bio-Rad protein assay kit. The total cell lysate $(20 \mu \mathrm{g})$ was loaded and separated on $6.0 \%$ SDS polyacrylamide gels. GSH-beads (AB Bioscience) were used for GST-pulldown assay using $700 \mu \mathrm{g}$ of total cell lysates. Transfer to PVDF membranes (Immobilon-P; Millipore) was carried out using a semidry transfer method (Trans-Blot; Bio-Rad) in $25 \mathrm{mM}$ Tris, $192 \mathrm{mM}$ glycine and $10 \%$ methanol for $1 \mathrm{~h}$ at $20 \mathrm{~V}$. Membranes were blocked in 5\% nonfat dried milk in Tris-buffered saline (TBS)/0.1\% Tween-20 and incubated with primary antibodies and horseradish peroxidase-conjugated secondary antibodies (Santa Cruz Biotechnology, Santa Cruz, CA) followed by enhanced chemiluminescence detection. Primary antibodies used in this study were anti-BAAT1 (Abcam, Cambridge, MA), anti-ATM (GeneScript, Piscataway, NJ), anti-DNA_PKcs (Calbiochem) anti-SMC1, anti-NBS1 and anti-GST (Santa Cruz Biotechnology). Additionally specific anti-phosphorylation antibodies were used against phospho-ATM (Ser1981; Cell Signaling, Danvers, MA), phospho-SMC1 (Ser966; Bethyl Laboratories, Montgomery, TX), phospho-NBS1 (Ser345) and phospho-DNA-PKcs (Ser2056) (Rockland). The anti-actin antibody (Santa Cruz Biotechnology) was used to validate the amount of protein. For immunoprecipitation, FLAG-tagged BAAT1 was expressed by transient expression. After the cells were lysed, anti-FLAG M2 beads (Sigma) were added to the lysates. The beads were extensively washed with lysis buffer and boiled at $95^{\circ} \mathrm{C}$. Following SDS-PAGE, the samples were transferred to membranes for immunoblot analysis as described above.

Mouse tissues were obtained from wild-type C57BL/6J mice (6-7 weeks old; Jackson Laboratory). Tissues were lysed using a homogenizer (200 series; Pro Scientific Inc., Oxford, CT) in ice-cold lysis buffer. Denatured proteins from the tissue

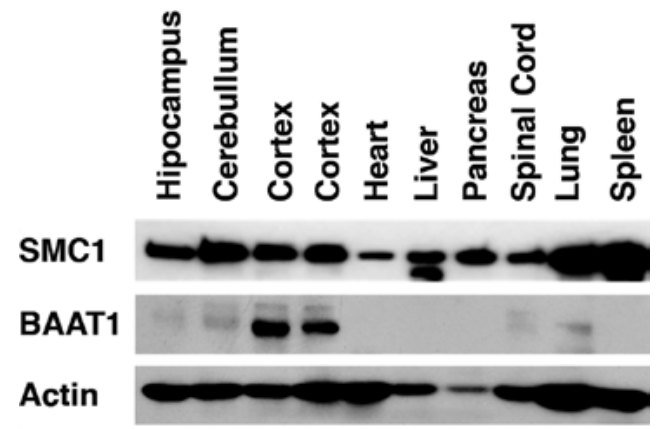

Figure 1. Tissue-specific expression of BAAT1. Samples from mice (6-7 weeks old) were lysed and immunoblotting was performed to detect the expression of BAAT1 and SMC1. Total lysates $(35 \mu \mathrm{g})$ for each tissue were studied. Actin was used as a loading control.

lysates were loaded at $35 \mu \mathrm{g}$ (total protein, Bradford assay) per lane on $8 \%$ SDS-PAGE gel and electro-blotted for $1 \mathrm{~h}$ at $25 \mathrm{~V}$ onto a PVDF membrane. Membranes were incubated for $1 \mathrm{~h}$ with $5 \%$ nonfat dry milk in TBST contaning $0.1 \%$ Tween- 20 to block non-specific antibody binding, and subsequently incubated with the primary antibodies for BRCA1, SMC1 and BAAT1. The antibody for actin was used to validate the amount of protein.

Construction of the GST-fusion protein. GST fragments were generated by PCR from human BAAT1 full-length plasmid containing amino acids 1-123 (\#1), 124-301 (\#2), 302-400 (\#3), 401-540 (\#4), 541-700 (\#5) and 701-821 (\#6), respectively. Segments of human BAAT1 were PCR amplified using the following primers: aa1-123, 5'-AA AGG ATC CAA ATG GAC CCAGAA TGC GCC CAG CTG-3' and 5'-AA AGC GGC CGC CTA CCA GCC GCT GCG CAC GGT GGG GAC-3'; aa124-301, 5'-CGC AGC GGC TGG ATC CAG GGC CTG CGC-3' and 5'-AA AGC GGC CGC CTA CCC CAA AGC CAG GGG TCC CAT GTG-3'; aa302-400, 5'-CTG GCT TTG GGG ATC CTG AAG CTC GAG-3' and 5'-AA AGC GGC CGC CTA GAG CCG CAG GAC AGT CAC TGT AGC-3'; aa401-540, 5'-AA AGG ATC CAA TGT GAC GGC TCG GCT GCC CCT GCC-3' and 5'-AA AGC GGC CGC CTA TGC GCA TCT GAA GTC AGC CTG TCC-3'; aa541-700, 5'-AA AGG ATC CAA CTC TTG GCT TCA GAG GTG CCT CAG-3' and 5'-AA AGC GGC CGC CTA GAG CCC CAC GTG GCA GAG AGC CCT-3'; aa701-821, 5'-AA AGG ATC CAA TTT GAC TTC GCC TTT TGT GCC TTG-3' and 5'-AA AGC GGC CGC TCA GTA GCA GTC GGC CTC GTC CCC-3'. The sequences of the amplified fragments were confirmed and subcloned into the pEBG vector (8).

Transfection. Transient transfection with a plasmid expressing GST-fusion proteins of BAAT1 was performed for $48 \mathrm{~h}$ with Lipofectamine 2000 (Invitrogen) according to the manufacturer's instructions, followed by NCS treatment. Transfection of BAAT1 siRNA was as described previously (7).

\section{Results and Discussion}

BAAT1 is highly expressed in the cortex. Recently, using the BRCT domain of BRCA1 as bait, our group isolated a cDNA 
A

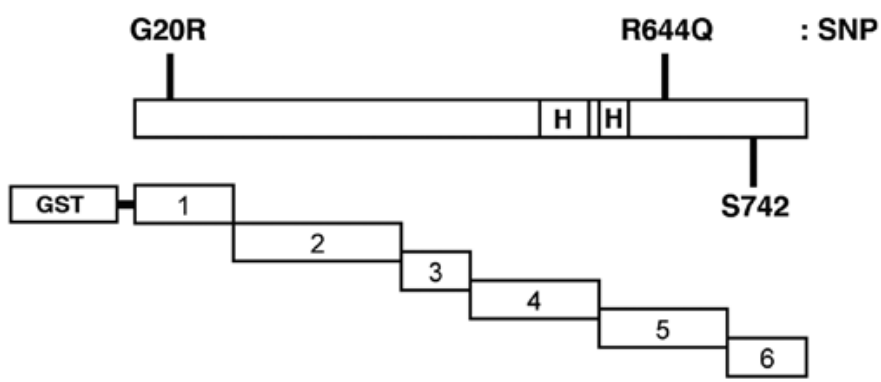

B

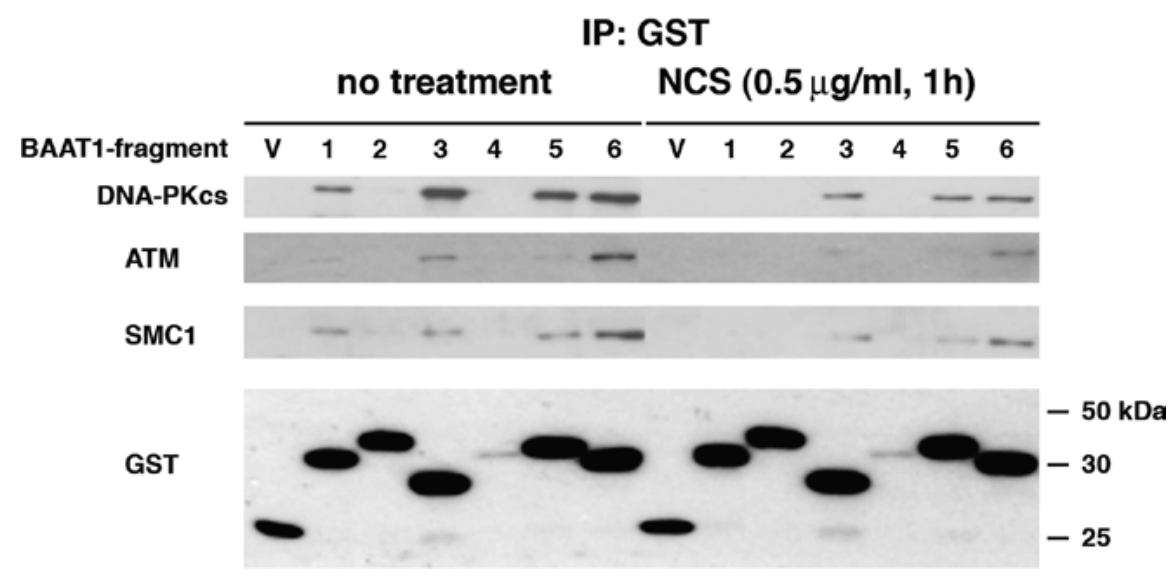

Figure 2. BAAT1 interactions with molecules related to DNA damage response. (A) BAAT1 structural features. Two SNP sites (G20R and R644Q), one putative phosphorylation site (S742) and two HEAT repeat domains (H) are shown. (B) Map of the regions of interaction. To determine the binding region of BAAT1, 293T cells were transfected with the pEBG2 vector (V) or plasmids containing GST-fusion BAAT1 fragments. After $48 \mathrm{~h}$, cells were treated with neocarzinostatin (NCS) or without. Total lysates were isolated and subjected to immunoprecipitation with GSH-sepharose beads as described previously (7). After immunoprecipitation, samples were immunoblotted with anti-DNA-PKcs, and protein levels were confirmed with anti-GST antibodies.

encoding a novel protein that we named BAAT1 (7). Northern blot analysis indicated that BAAT1 mRNA is ubiquitously expressed, and a much higher mRNA level is detected in testis (7). In the present study, levels of BAAT1 protein were assessed using the samples obtained from mouse tissues. As shown in Fig. 1, high levels of BAAT1 protein were detected in the cortex. Much lower levels of this protein were detected in the cerebellum, spinal cord and lung. SMC1 was detected in all of the tissues studied, although the protein was differentially expressed in these tissues. These results suggest that BAAT1 is involved in the neuronal system.

BAAT1 binds to ATM, DNA-PKCs and SMCl. ATM is phosphorylated at Ser1981 when cells are exposed to cell stress (9). In BAAT1-depleted cells, this phosphorylation of ATM at Ser1981 was greatly reduced, indicating that BAAT1 is required for ATM activation. We further explored whether BAAT1 is involved in other pathways.

The full-length BAAT1 protein consists of 821 amino acids of $\sim 88 \mathrm{kDa}$, and the human amino acid sequence shares 73.5 and $72.9 \%$ identity with mouse and rat sequences, respectively. As shown in Fig. 2A, the C-terminal half of the protein contains two HEAT (Huntingtin, elongation factor 3 , A subunit of protein phosphatase $2 \mathrm{~A}$ and TOR1) repeat domains (aa495-531, aa544-576) and a putative phosphorylation residue at amino acid Ser742. From the database, human BAAT1 has two SNP sites (G20R and R644Q), although it is not known whether these SNPs are pathogenic. Six fragments of BAAT1 protein were expressed with an N-terminal GST tag in the 293 T cells (Fig. 2A). After 48 h, the cells were treated with NCS for $1 \mathrm{~h}$ to induce DNA DSBs. Each of the GST fragments were pulled down, and the interaction with cellular proteins was assessed. Immunoblotting with an anti-GST antibody revealed the protein levels of the GST-\#4 segment of BAAT1 were lower than the others that were found to be similarly expressed, suggesting that GST-\#4 is unstable.

Immunoblot analysis demonstrated that both the DNA-PKcs and SMC1 bound to BAAT1 fragments \#1, \#3, \#5 and \#6 before NCS treatment, while interaction with \#1 was decreased after NCS treatment. ATM bound to the \#1, \#3 and \#6 fragments of BAAT1, although its interaction with BAAT1 \#1 was much weaker than with the others. After NCS treatment, ATM bound primarily to the BAAT1 \#6 fragment, although BAAT1 \#3 also showed weak interaction. Interaction between ATM and BAAT1 \#1 was not detected after NCS treatment. These results suggest that protein modification and/ or allosteric change in the conformation that occurs in BAAT1, DNA-PKcs, ATM and/or SMC1 after NCS treatment determines the intensity of the interaction among these proteins. We also examined physical interaction between BAAT1 and $\mathrm{Ku} 70 / \mathrm{Ku} 80$, which are components of the DNA-dependent protein kinase, but co-immunoprecipitation was not detected (data not shown).

Time-dependent interaction between BAAT1 and ATM/ $D N A-P K c s$. As shown in Fig. 2, the intensity of the interaction 
IP: FLAG

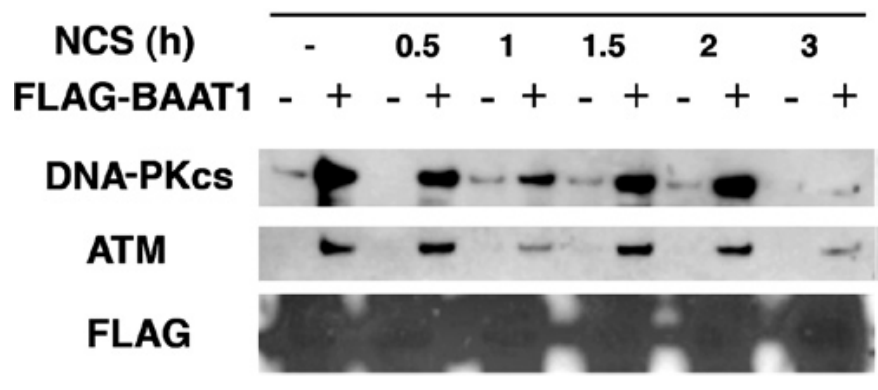

Total lysate

$\begin{array}{llllll}- & 0.5 & 1 & 1.5 & 2 & 3\end{array}$
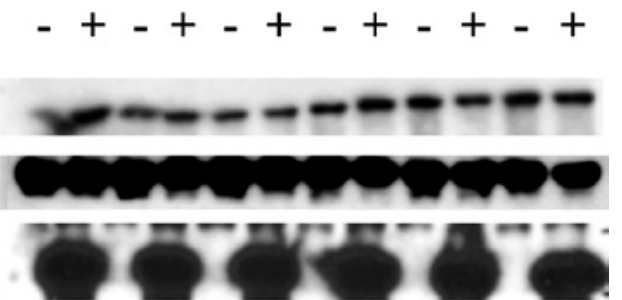

Figure 3. Time-dependent interaction between BAAT1 and ATM/DNA-PKcs after treatment with NCS. After 48 h, cells were treated with NCS for the indicated times. Total lysates were prepared and immunoprecipitated with the anti-FLAG antibody. After immunoprecipitation, samples were blotted with the indicated protein antibodies.

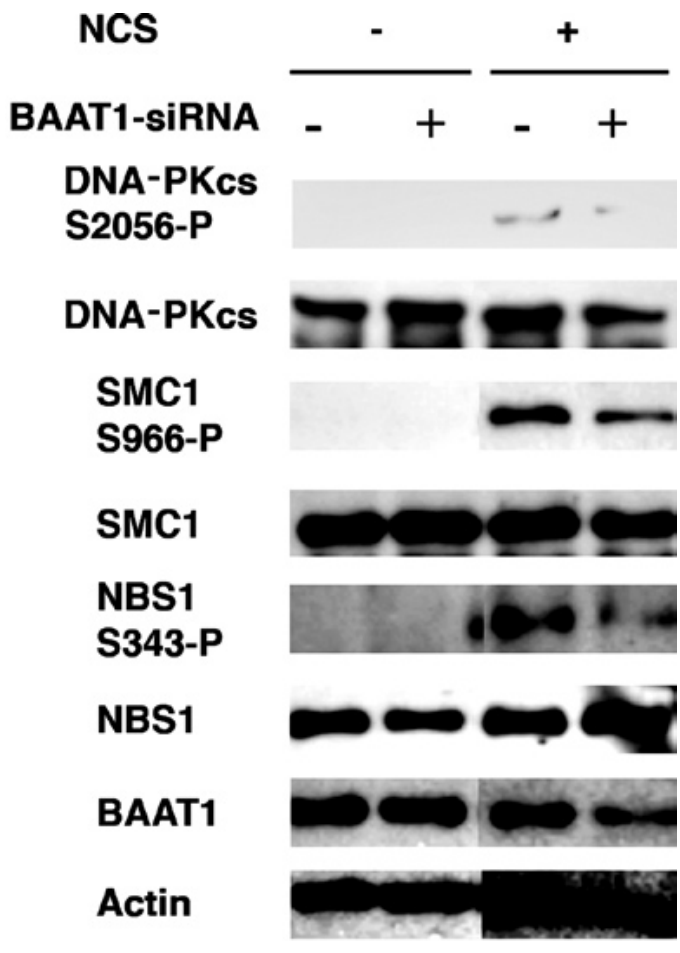

Figure 4. Down-regulation of BAAT via siRNA. MCF10A normal human mammary epithelial cells were transfected with siRNA oligomer for BAAT1 knockdown as previously described (7). After $72 \mathrm{~h}$, cells were treated with NCS for the indicated times. Total lysates were prepared and subjected to Western blotting. The level of total protein and phosphorylation by NCS were detected with the indicated antibodies. Actin protein was used as a loading control for immunoblotting.

between BAAT1 and other cellular proteins may be regulated by DNA damage stimuli. We assessed the interaction of BAAT1 and ATM or the DNA-PKcs with NCS treatment in a time-dependent manner. Cells (293T) were transfected with FLA-tagged BAAT1 for $48 \mathrm{~h}$ and treated with NCS for $0.5,1$, 1.5, 2 and 3 h. FLAG-BAAT1 was pulled down by anti-FLAG beads, and samples were immunoblotted with anti-DNA-PKcs or -ATM antibodies (Fig. 3).

Levels of endogenous DNA-PKcs, ATM and exogenous FLAG-BAAT1 were not altered throughout this time course. After FLAG-BAAT1 was pulled down, interaction with both the DNA-PKcs and ATM was detected, but was decreased after $1 \mathrm{~h}$. Interaction was then recovered $1.5 \mathrm{~h}$ after NCS treat- ment, and decreased significantly after $3 \mathrm{~h}$. The mechanism involved in this biphasic alteration of interaction after NCS treatment remains to be elucidated.

BAAT1 knockdown reduces phosphorylation of the DNA-PKcs at Ser2056 and SMC1 at Ser966 by NCS. Since siRNA-mediated depletion of BAAT1 was previously found to impair the phosphorylation of ATM at Ser1981 after IR treatment (7), we investigated whether it also regulates the phosphorylation of the DNA-PKcs and SMC1. The normal mammary epithelial cell line MCF10A was transfected with control or BAAT1 siRNA for $48 \mathrm{~h}$, followed by NCS treatment.

Chen et al found that IR induces the autophosphorylation of the DNA-PKcs at Ser2056, which is required for the repair of DSBs by non-homologous end joining (10). As shown in Fig. 4, NCS treatment induced the autophosphorylation of the DNA-PKcs at Ser2056, however, this phosphorylation was significantly reduced when BAAT1 was knocked down by siRNA.

SMC1 proteins plays a pivotal role in sister chromatid cohesion, chromosome condensation, gender-chromosome dosage compensation, and DNA recombination and repair (11-14). Heterodimers of the SMC1 and SMC3 proteins have been implicated specifically in both sister chromatid cohesion and DNA recombination. ATM phosphorylates SMC1 protein at Ser957 and Ser966 after IR, and expression of a phospho-deficient form of SMC1 protein mutated at these phosphorylation sites abrogates IR-induced $\mathrm{S}$ phase cell cycle checkpoints $(15,16)$. In the present study, when cells were treated with NCS, the phosphorylation of SMC1 at Ser966 was significantly increased, while this phosphorylation was reduced in BAAT1-knockdown cells. As shown previously (7), phosphorylation of NBS1 at Ser343 was also decreased in BAAT1-knockdown cells.

There are several possible mechanisms involved in the decreased phosphorylation of DNA-PKcs, SMC1 and NBS1 in BAAT1-knockdown cells: i) kinase activity responsible for phosphorylation is inhibited, ii) phosphatase activity is increased, or iii) localization of DNA-PKcs to the sites of DSBs is inhibited. Although our previous studies have suggested that BAAT1 may regulate ATM phosphatase (7), we are also currently investigating other potential mechanisms using human cell lines in which BAAT1 is stably knocked down or knocked out (unpublished data). 
As described above, BAAT1 has two tandem copies of the HEAT repeat, which may be important for protein-protein interaction. Two SNP sites in the coding sequence have been reported, although it is not clear whether these mutants are pathogenic. To elucidate the exact function of BAAT1 in the DNA damage response and cell cycle checkpoints, structural integrity should also be investigated.

To date, approaches to determining the role of BAAT1 in the DNA damage response have involved the evaluation of the binding affinity and the modulation of phosphorylation of the proteins involved in DNA damage signaling using cells in which BAAT1 is suppressed by knockdown. To elucidate more of the physiological roles of these proteins, the generation of knockout mice is essential.

\section{Acknowledgements}

The authors would like to acknowledge Dr Ruth Seal (HUGO Gene Nomenclature Committee) for specific suggestions and registration of BAAT1/BRCA1-associated ATM-activator 1 in the Human Genome Organization (HUGO) database. The authors also thank the members of the Ouchi laboratory for the helpful discussion. This study was supported by R01CA79892 and R01CA90631 (both from NIH/NCI to T.O.), the AVON pilot project grant (to T.O.) and Ms. Susan G. Komen, Investigator Initiated Research Grant (to T.O.).

\section{References}

1. Mahaney BL, Meek K and Lees-Miller SP: Repair of ionizing radiation-induced DNA double-strand breaks by non-homologous end-joining. Biochem J 417: 639-650, 2009.

2. Pandita TK and Richardson C: Chromatin remodeling finds its place in the DNA double-strand break response. Nucleic Acids Res 37: 1363-1377, 2009.
3. Lee SH and Kim CH: DNA-dependent protein kinase complex: a multifunctional protein in DNA repair and damage checkpoint. Mol Cell 13: 159-166, 2002.

4. Shiloh Y: ATM and related protein kinases: safeguarding genome integrity. Nat Rev Cancer 3: 155-168, 2003.

5. Uziel T, Lerenthal Y, Moyal L, Andegeko Y, Mittelman L and Shiloh Y: Requirement of the MRN complex for ATM activation by DNA damage. EMBO J 22: 5612-5621, 2003.

6. Tischkowitz MD and Hodgson SV: Fanconi anaemia. J Med Genet 40: 1-10, 2003.

7. Aglipay JA, Martin SA, Tawara H, Lee SW and Ouchi T: ATM activation by ionizing radiation requires BRCA1-associated BAAT1. J Biol Chem 281: 9710-9718, 2006.

8. Ouchi, T, Lee SW, Ouchi M, Aaronson SA and Horvath CM: Collaboration of STAT1 and BRCA1 in differential regulation of IFNg target genes. Proc Natl Acad Sci USA 97: 5208-5213, 2000.

9. Bakkenist CJ and Kastan MB: DNA damage activates ATM through intermolecular autophosphorylation and dimer dissociation. Nature 421: 499-506, 2003.

10. Chen BP, Chan DW, Kobayashi J, Burma S, Asaithamby A, Morotomi-Yano K, Botvinick E, Qin J and Chen DJ: Cell cycle dependence of DNA-dependent protein kinase phosphorylation in response to DNA double strand breaks. J Biol Chem 280: 656-662, 2005.

11. Strunnikov AV and Jessberger R: Structural maintenance of chromosome (SMC) proteins: conserved molecular properties for multiple biological functions. Eur J Biochem 263: 6-13, 1999.

12. Losada A and Hirano T: Dynamic molecular linkers of the genome: the first decade of SMC proteins. Genes Dev 19: 1269-1287, 2005.

13. Watrin E and Peters JM: Cohesin and DNA damage repair. Exp Cell Res 312: 2687-2693, 2006

14. Wong RW: An update on cohesin function as a 'molecular glue' on chromosomes and spindles. Cell Cycle 9: 1754-1758, 2010

15. Kitagawa R, Bakkenist CJ, McKinnon PJ and Kastan MB: Phosphorylation of SMC1 is a critical downstream event in the ATM-NBS1-BRCA1 pathway. Genes Dev 18: 1423-1438, 2004.

16. Yazdi PT, Wang Y, Zhao S, Patel N, Lee EY and Qin J: SMC1 is a downstream effector in the ATM/NBS1 branch of the human S-phase checkpoint. Genes Dev 16: 571-582, 2002. 